\section{Enter the anode matrix}

\section{ce}

Because we

have a host to enable lithium stripping and deposition within the framework, there is no significant electrodelevel volume expansion
By infusing lithium into a carbon scaffold, Yi Cui and co-workers at Stanford University have established a simple means of preventing volume expansion and dendrite formation in lithium-metal batteries.

A lithium-metal anode is considered to be the 'holy grail' by many in the battery community: it has a tenfold higher theoretical capacity than graphite - the industry standard in lithium-ion batteries. It is also lightweight and has the lowest anode potential of all known electrode materials. However, safety and performance issues associated with an unstable interface and lithium dendrite formation must be overcome for lithium-metal battery technologies to be commercially viable.

Previous attempts to mitigate dendrite growth have focused on stabilizing the solid-electrolyte interphase (SEI) with additives or by constructing an artificial SEI. "In the past, we have tried to use nanoscale interfacial engineering, with hollow carbon spheres, boron nitride and graphene, to build a stable interface and to control lithium deposition," says Cui. Although such approaches are promising, they ignore a crucial failure mechanism - the extreme volume expansion that the 'hostless' electrode undergoes upon lithiation.

As they report in Proceedings of the National Acadamy of Sciences USA, the researchers introduce the concept of encapsulating lithium in a scaffold matrix as a new way to tackle dendrite formation and volume fluctuation. As Cui explains, "we recognized that the root cause of lithium-metal dendrite formation and instability of the interface is the infinite relative volume expansion due to the lack of a host".
A suitable host material for lithium needs to be chemically and mechanically stable, lightweight (to avoid reducing the gravimetric density of lithium in the composite), have good ionic and electronic conductivity, and have a large surface area. Taking these requirements into consideration, the researchers opted for an electrospun carbon-fibre network. To entrap lithium, they developed a melt-infusion method, whereby molten liquid lithium infuses into the 3D scaffold and then solidifies with a porous morphology in the spaces between the fibres. Although uncoated carbon fibres are 'lithiophobic' (that is, they are not wet by liquid lithium, which results in poor infiltration), coating the fibres with silicon makes the scaffold 'lithiophilic', and allows excellent infusion of lithium throughout the framework.

Owing to the high conductive surface area, stable SEI and negligible volume fluctuation, the composite lithium/carbon electrode could be stably cycled at high current density, in stark contrast with its bare-metal counterpart. According to Cui: "The most significant finding is that because we have a host to enable lithium stripping and deposition within the framework, there is no significant electrode-level volume expansion." Not only is the risk of failure greatly reduced by the mechanical stability of this composite electrode, but the general approach may be extended to other metal-anode battery systems in the future. Despite the improved electrochemical performance, further improvements to this design are required. In particular, Cui points out, "the path forward is to build a more stable interface around the host that reduces side chemical reactions between lithium metal and the electrolyte".

Adam Brotchie

ORIGINAL ARTICLE Zheng, L. et al. Composite lithium metal anode by melt infusion of lithium into a 3D conducting scaffold with lithiophilic coating. Proc. Natl Acad. Sci. USA http://dx.doi. org/10.1073/pnas.1518188113 (2016)

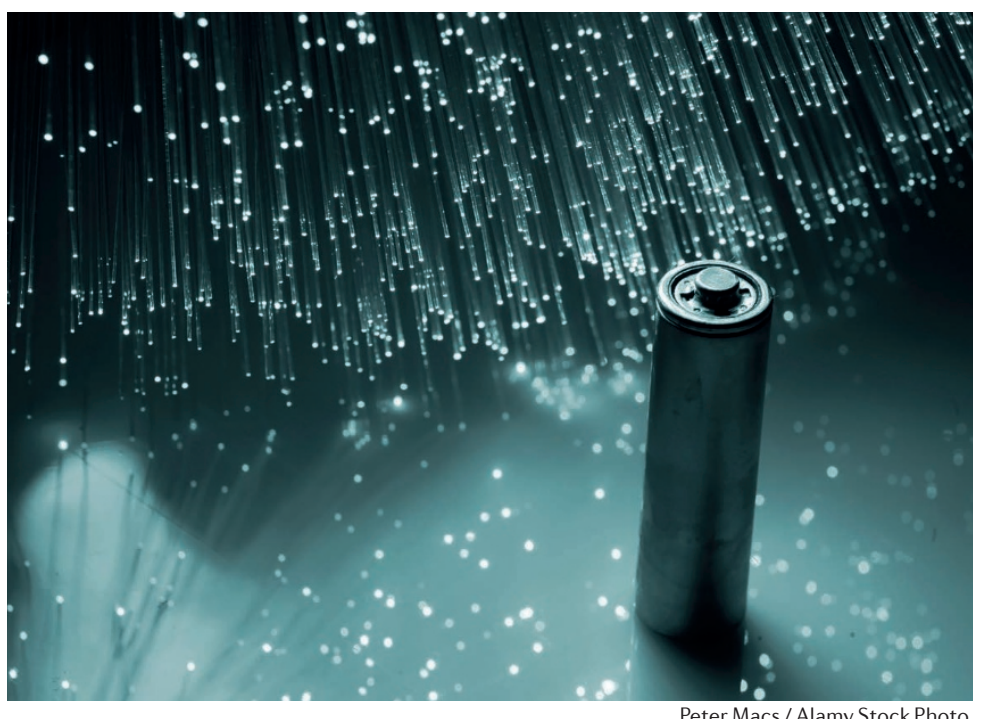

